\title{
Smart object reminders with RFID and mobile technologies
}

\author{
Hui-Huang Hsu ${ }^{\mathrm{a}, *}$, Cheng-Ning Lee ${ }^{\mathrm{a}}$, Jason C. Hung ${ }^{\mathrm{b}}$ and Timothy K. Shih ${ }^{\mathrm{c}}$ \\ ${ }^{a}$ Department of Computer Science and Information Engineering, Tamkang University, 151 Ying-Chuan \\ Rd., Tamsui, New Taipei City, Taiwan \\ ${ }^{\mathrm{b}}$ Department of Information Management, Overseas Chinese University, 100 Chiao Kwang Rd., \\ Taichung, Taiwan \\ ${ }^{\mathrm{c}}$ Department of Computer Science and Information Engineering, National Central University, 300 \\ Jhongda Rd., Jhongli, Taoyuan, Taiwan
}

\begin{abstract}
In this paper, we present a reminder system that sends a reminder list to the user's mobile device based on the history data collected from the same user and the events in the user's calendar on that day. The system provides an individualized service. The list is to remind the user with objects he/she might have forgotten at home. The objects that the user brings along with are detected by passive RFID technology. Objects are classified into three different levels based on their frequencies in the history data. Rules of the three levels are then followed to decide if a certain object should be in the reminder list or not. A feedback mechanism is also designed to lower the possibility of unnecessary reminding.
\end{abstract}

Keywords: RFID, reminder systems, personalization, smart home, ambient intelligence

\section{Introduction}

Sensors are the basic components needed to detect the contextual needs of the users. In recent years, sensor network research has been a hot topic [1-3]. In a smart home, sensors are deployed at home to collect user-centered information in the environment. The collected information is then processed by the server to provide suitable services to the user. This is also a part of ambient intelligence. Some people tend to forget things when they leave home for work or school. It is desirable that people are reminded with the things they forgot to bring along with. In this paper, we propose a smart reminding mechanism using RFID (Radio Frequency Identification) and mobile technologies. The mechanism is designed to fit individual needs from the history data and the event calendar of the user.

Previously, RFID was used for abnormal behavior detection in elderly care [4] and object reminders to school kids [5]. According to the survey of IDTechEx, the applications of RFID will reach the highest demand from 2015 to 2020 . Although the current RFID software market is not so big, with the growing popularity of RFID the RFID software market will have a potential high growth in the near future [6]. Research in location-based queries in a mobile environment can be found in [7,8]. For further researches on mobile services, the readers are referred to [9-11].

\footnotetext{
${ }^{*}$ Corresponding author. Tel.: +886 226215656 Ext. 3307; Fax: +886 2 26209749; E-mail: h_hsu@mail.tku.edu.tw.
} 
In smart home research, Future Home is a real environment built by Microsoft [12]. It includes the front door, the entry/foyer, the kitchen, the family room, the dining room, the entertainment room and the bedroom. In Japan, Toyota also built a future home called PAPI [13]. The research combined automatic cars and IT facilities produced by Toyota. In Taiwan, Farglory Realty used advanced IT technologies for home security, such as face recognition and finger vein identification.

This research aims at providing a reminding mechanism for the user in a smart home environment. We focus on objects that the user would bring along when he/she goes out. The RFID technology is used to sense the objects the user brings along at the front door. In the object database, an object is recorded not only by its name along with a unique RFID number, but also its class. The taxonomy has two levels: classes and objects. For example, both the June issue and the July issue of Scientific American have the same class - Scientific American. Furthermore, both classes and objects are classified into three different levels according to the frequencies the user took them out. The three levels are daily, weekly and non-regular. The reminder system is also connected to the user's calendar. Objects related to events on the calendar are also recorded in the database. An event object list can be built by the user or automatically detected and recorded by the system.

When the user leaves home, the reminder system checks the objects in his/her bags and pockets, and compares the objects with a list of objects generated by the system according to the day of the week and the events on the calendar. The system then sends a reminder object list to the mobile phone or PDA (Personal Digital Assistant) of the user. The user can quickly browse the list and take any objects he/she might have forgotten to bring along. There is also a feedback mechanism for the reminder list. If any objects mistakenly appear on the list, the user can give a feedback to the system. The user can simply mark the unneeded objects on the reminder list. Those objects will not be included in the reminder list in the same situation next time. In this research, we have implemented a prototype system for the ideas. The preliminary tests show that this approach is promising for real applications.

In Section 2, related work in RFID applications and smart environment is discussed. The proposed smart reminding mechanism is introduced in Section 3. System implementation and tests are then presented in Section 4. Finally, a brief conclusion is drawn in Section 5.

\section{Related work}

\subsection{RFID applications}

There are three major components of an RFID system: the reader, the antenna, and the tags. Each tag is associated with a unique number. When a tag is in the detection range of the reader, the number is read. Two types of tags can be found: active tags with a longer detection range and passive tags with a shorter detection range [14]. An RFID tag is usually attached to an object and the information of the object along with the RFID number are recorded in the database. Whenever the RFID tag is sensed, the object can thus be identified.

The papers to be discussed here are more advanced applications. One of the papers deploys a large amount of RFID tags in an office, a conference venue or other public places. The user can use mobile devices to receive desired information like locations or maps, also the user can leave a message to a certain person. Besides, the maps can be retrieved by the rescue crew in emergency through a tag [15]. RFID was also applied to home cooking. First, RFID tags are attached to ingredients and utensils. The system can automatically provide video instructions to the user according to the detected movements of the user. The user needs not check the cook book step by step [16]. 
Another paper proposed a client-server architecture that can remotely control home appliances via mobile devices. Massive RFID tags are distributed in the environment for location awareness. The advantages of this system are less power consumption and design complexity [17]. In medical care, there is an RFID application related to medicine taking. RFID tags are attached to medicine bottles and the reader is placed in the drawer storing the medicine. The system can help the elderly people to record their medicine-taking data and determine whether they have taken the right medication with right dosage [18]. On the other hand, the assessment of independent living ability for the elderly can also be done by the RFID technology. RFID tags are attached to the tools used in our daily life. Readers are placed on different body parts of the elder. Home activities of the elder can thus be recorded $[19,20]$. Above-mentioned researches were keen to use the RFID technology to provide the users with convenient services. Although the RFID tags deployed in the public areas can help reach the goal, the tag price is still too high to make it feasible in real applications. In addition, using RFID to detect user's activities requires the user to carry RFID readers. This is very inconvenient to the user. Hopefully, the RFID reader can become much smaller and light-weighted in the near future.

\subsection{Smart environment}

According to Mark Weiser, there would be many invisible sensors, actuators, computers and displays, embedded into our daily lives and contacted each other via the Internet in the real world. Smart environment is defined to be a small world with numerous smart devices constantly working to provide comfort to the inhabitants [21-23]. This concept is commonly used in residential. Researchers hope to use machine learning methods to learn the living habits of each inhabitant, and intelligent agents to provide services to each user. Generally speaking, these studies have to collect enough information/data first to train the machine learning system. For example, inhabitant behaviors at home can be recorded with a time stamp. The relationship of the behaviors in time can thus be analyzed [24-26]. Another issue is that people's behavior and demand usually change with time. Therefore, the system must also adapt to each user. A research used cameras, microphones and other wearable sensors to track the inhabitant. The support vector machine (SVM) was then used to determine the user's behavior change and hence different environment settings [27]. A good system not only can changes with the user, but also can predict the next user activity. Another research focused on patients with dementia and loss of short-term memory. A system was designed to remind the patients if a certain action has been taken (such as having tea, brushing teeth, and... etc.). When the patient forgets the next action, the system can give tips through LED lights, images or text to remind the patient [28].

\section{Smart reminding mechanism}

A smart reminding mechanism is designed to remind the user what he or she has forgotten to bring along when leaving home for work or school. The user can thus takes the needed objects before going far from home. The RFID technology is used here to sense the objects. There are two ways to determine the list of objects a person needs for daily work. First, if the events on that day are known, objects for those events should be in the list. Secondly, if the objects that the user used to bring along with on that certain day of the week, they should also be in the list. In this research, we tried to combine these two sources of information to construct the reminder list for the user when he/she goes out for work or school on a certain day. 


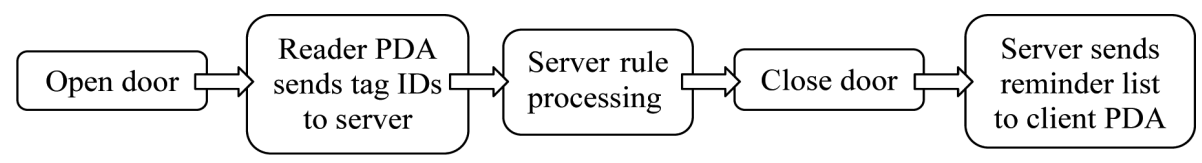

Fig. 1. Workflow of the reminder system.

The background setting of the developed system is in a general home environment. An RFID reader is installed near the front door and RFID tags are attached to the objects the user would take out. Whenever the user goes out, the objects he/she brings along with are detected by the reader and saved in the database. In general, the objects are strongly related to the day in a week. For example, Jack is a student and he has Calculus classes on Wednesday. Therefore, he usually brings the Calculus textbook with him on Wednesday. Max plays tennis with his friends on Friday evening. He brings his tennis racquet with him on Friday.

\subsection{Reminder server}

Figure 1 illustrates the workflow of the reminder system. When the front door is open, the RFID reader is triggered to read the nearby tags and send their ID numbers to the server. The maximum detection distance should be set at around 1 meter. The server compares the list with the object list generated by a number of rules following the historical data and the calendar events. The missing objects are arranged in a reminder list. When the door is closed, the server sends the list to the mobile phone or PDA of the user. The functions of the reminder server and the PDA client are discussed in the following.

(a) Collect tag IDs from the RFID reader.

(b) Process the rule sets.

(c) Generate and send the reminder list to the PDA client.

The user needs to initiate the system by inputting the object data including the object name, its RFID tag ID, and its class to the object database in the server. Recall that the objects are placed in a two level taxonomy: classes and objects. When the server received the tag IDs from the reader (detected when the user leaves home), it records the date along with the tag IDs. The tag IDs are associated with the objects and their classes in the database. The object/class use records for a week are then processed by the rule sets discussed in the following.

There are three sets of rules: the object rules, the class rules and the event rules. The object rules are used to classify all objects into one of the three frequency levels: daily, weekly and non-regular (Table 1). Daily means that the object is taken out by the user every day, e.g., keys and wallet. Weekly means that an object is needed on certain days during a week, e.g., English textbook on Monday and Wednesday because there are English classes on the two days. The days can be found from the use records in the previous week and they are recorded with the object in the database. As for non-regular, the objects have not been detected in the past seven days. The rules also define the conditions for object level change. An object can be upgraded or downgraded in the three levels. The adjustment periods of upgrade and downgrade can be made daily or weekly. For daily checks, the system makes adjustments right after the user leaves home every day. For example, if a non-regular object is brought by the user today, it is upgraded to a weekly object right away. If a weekly object is not brought by the user today, it is downgraded to a non-regular object. For weekly checks, the rules are performed every seven days. For example, if a weekly object was taken every day in the past week, it is upgraded to a daily object. If a daily object was not taken in any days in the past week, it is downgraded to a weekly object. 
Table 1

Rules for object classification, upgrade and downgrade

\begin{tabular}{|c|c|c|c|}
\hline Object classification & Classification condition & Upgrade condition & Downgrade condition \\
\hline Daily & $\begin{array}{l}\text { Object is brought every } \\
\text { day in the past week. }\end{array}$ & Not applicable & $\begin{array}{l}\text { Object is not brought for } \\
\text { some days in the past week. } \\
\text { (Check every week) }\end{array}$ \\
\hline Weekly & $\begin{array}{l}\text { Object is brought } 1 \text { to } 6 \\
\text { days in the past week. }\end{array}$ & $\begin{array}{l}\text { Object is brought every day } \\
\text { in the past week. } \\
\text { (Check every week) }\end{array}$ & $\begin{array}{l}\text { Object is not brought in } 7 \\
\text { consecutive days. } \\
\text { (Check every day) }\end{array}$ \\
\hline Non-regular & $\begin{array}{l}\text { Object is not brought in } \\
\text { the past } 7 \text { days. }\end{array}$ & $\begin{array}{l}\text { Object is brought today. } \\
\text { (Check every day) }\end{array}$ & Not applicable \\
\hline
\end{tabular}

The second rule set is called class rules. A class is a collection of objects with similar features. For example, different issues of the same magazine are of the same class. Classes are also classified into daily, weekly and non-regular and the object classification rules in Table 1 are applicable to the classes. Therefore, the reminder list generated by the server includes not only the objects, but also the classes. For example, if a user takes Business Week with him/her every day, it is certain that the system will remind him/her whenever he/she forgets to bring any issue of Business Week on that day. Moreover, for the same medicine, every bottle has its unique tag ID. The user might need the medicine every day, but he/she might bring different bottles. If all the bottles are set to the same medicine class, the system can remind the user by the medicine class, not a particular bottle. The upgrade and downgrade rules in Table 1 also apply to the classes, but they are processed only in a weekly basis.

The third rule set is event rules. The rules help record the objects a user needed for a certain event. The objects for an event can be determined by the historical data and adjusted by the user through the feedback mechanism or they can be input directly by the user. When there is an event today and the event objects do not exist in the database. The event objects can be determined by subtracting daily and weekly objects from the objects that the user brings with him/her today. The server will automatically add the objects to the event. This can be illustrated by the following equation: Event_Objects $=$ All_Objects Daily_Objects - Weekly_Objects. If there are more than one unknown events, this might cause incorrect event object lists. However, they can be corrected with the user feedback at a later time. This process is shown in the upper half of the flowchart in Fig. 2. The object rules and the class rules are performed in the lower half of the flowchart.

Finally, a reminder list is sent to the user's PDA or smart phone before he or she goes far. The objects in the reminder list are ordered as follows: 1) event objects, 2) daily objects, 3) weekly objects, 4) daily classes, and 5) weekly classes. Meanwhile, level upgrade and downgrade rules are applied and related records are updated.

\subsection{PDA client}

A PDA is used in our research as the client. A smart phone is another possibility. There are three main functions of the client.

(a) Reminder: When the user leaves home, the system automatically compares the taken objects with the object list generated by the system. The system then sends the reminder object/class list to the user's PDA. The PDA client is responsible for displaying the list to the user.

(b) Calendar: The user can manage events in his/her personal calendar on the PDA. The system will automatically update the calendar of the user on the server. 


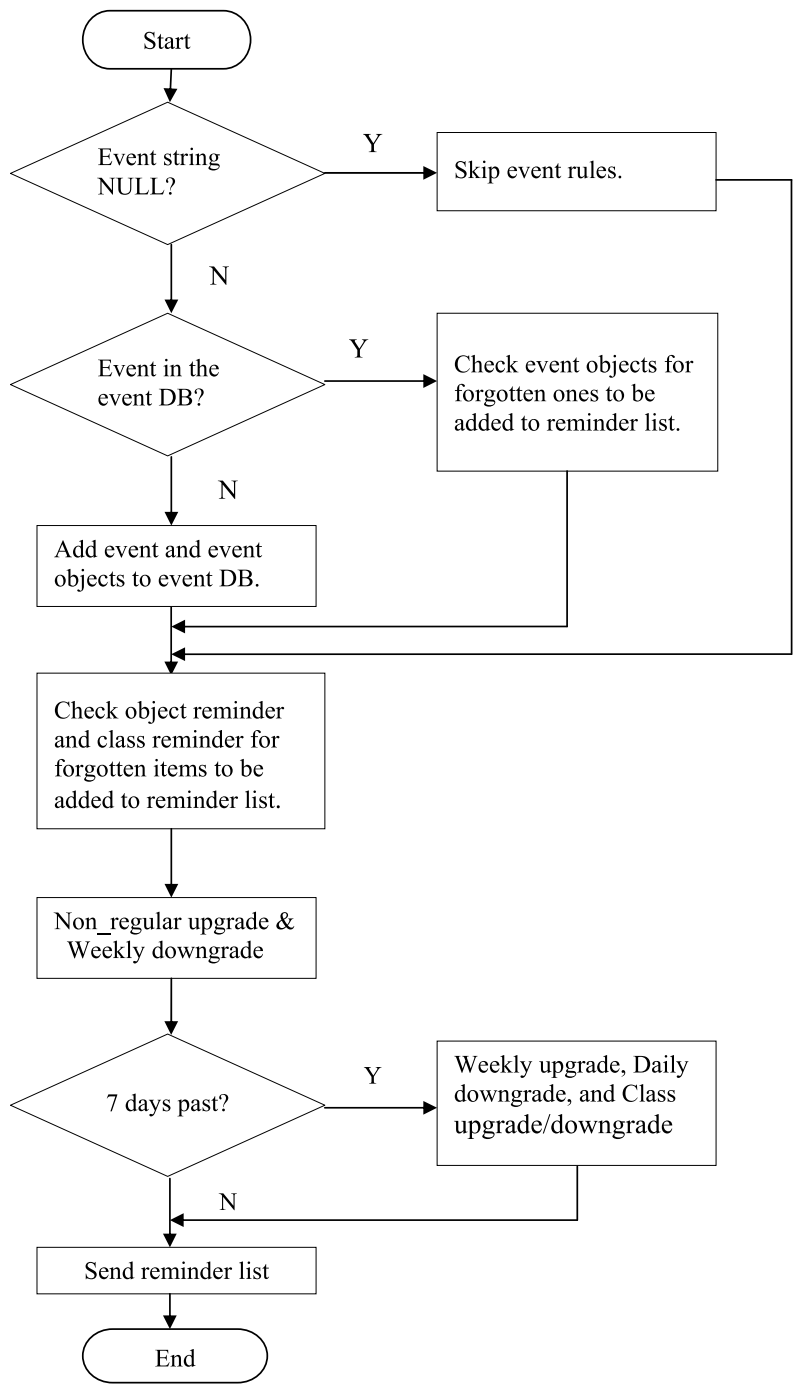

Fig. 2. The flow chart of rule processing.

(c) User feedback: If the user is not satisfied with the reminder list, he/she can decide whether to ignore some of the objects in the reminder list and give a feedback to the system. The object/class frequency levels are then adjusted accordingly. Also, the user can modify the event objects for each event. With this feedback mechanism, the reminder system can provide a better service to the user with a more accurate reminder list.

\section{System implementation and tests}

Passive tags are first attached to the objects in our implementation. They are used, rather than active tags, because detection range is more appropriate for this application. Also, passive tags are smaller and lighter than active tags. Figure 3 shows a few examples. The RFID tags follow the ISO15693 


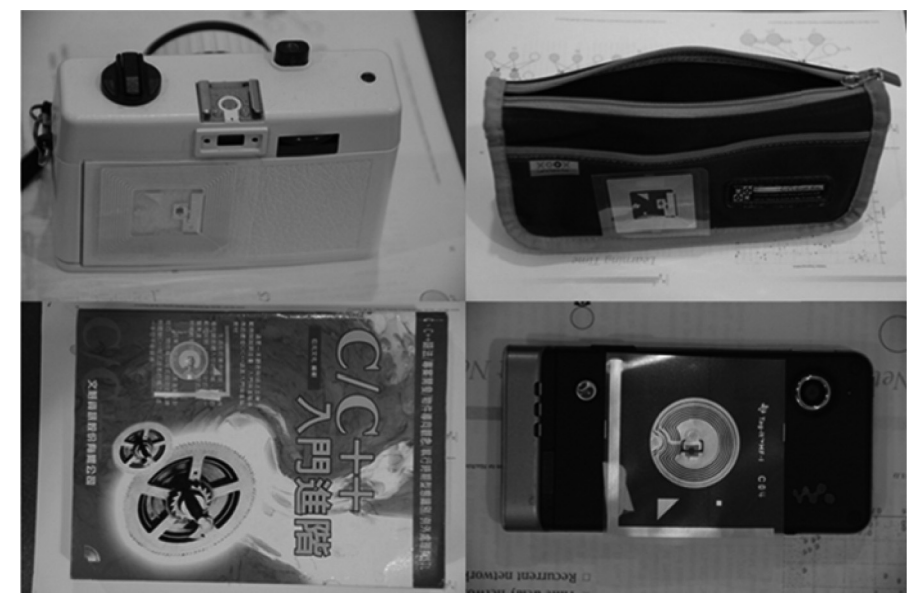

Fig. 3. Objects attached with an RFID tag.

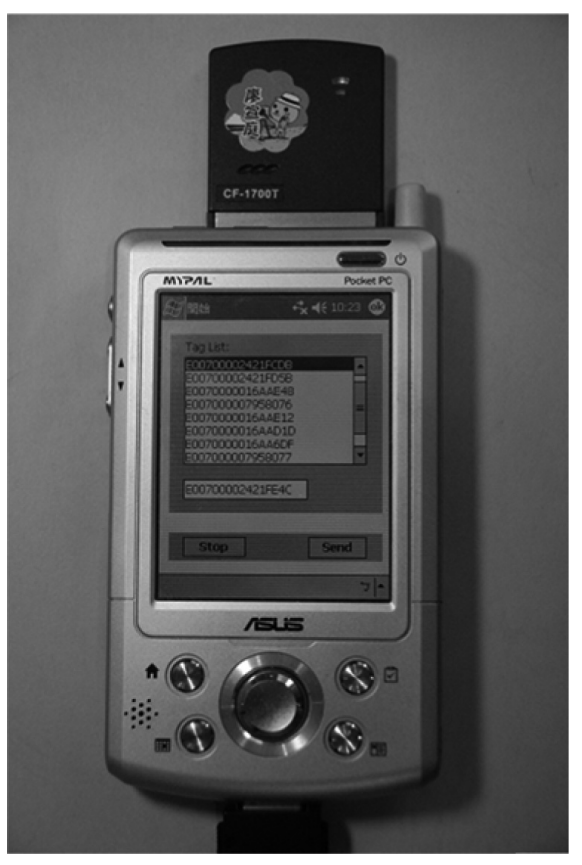

Fig. 4. The CF-card RFID reader mounted on he reader PDA.

standard. They can be detected by the reader with a reading/detection distance of 10 centimeters. An RFID reader with a CF (Compact Flash) transmission interface was used in this research. The reader model is CF-1700T by Sunion (http://www.sunion.com.tw/) with a working frequency at $13.56 \mathrm{MHz}$. The reader needs to be attached to a PDA with a CF slot. The power of the reader is supplied by the PDA with a consumption rate at $15 \mathrm{~mA}(\max )$ in operation and $4 \mathrm{~mA}$ in idle. The PDA used for the RFID reader is of ASUS A716 Series with an Intel Xscale PXA255 $400 \mathrm{MHz}$ processor. It runs on a Microsoft Windows Powered Pocket PC 2003. The PDA has a CF Type II slot to plug-in the RFID reader and can access wireless LAN through WiFi (Fig. 4). 


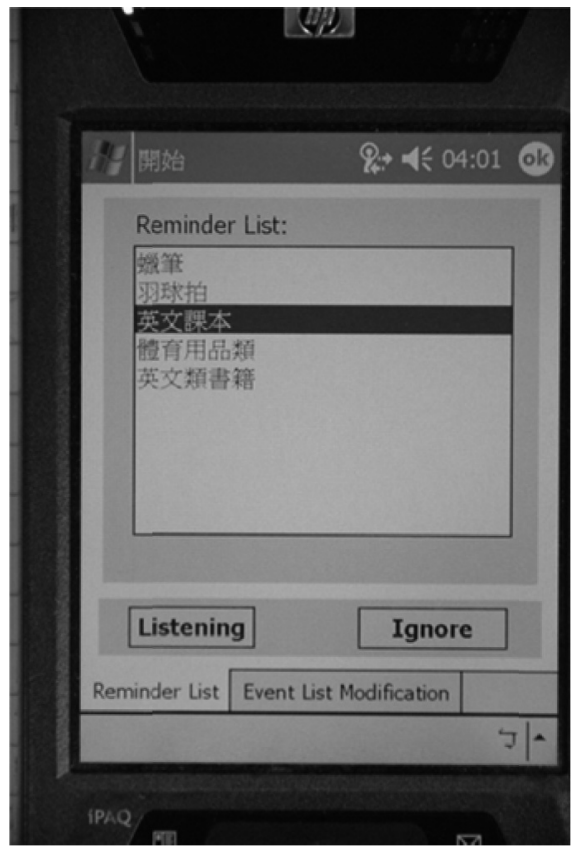

Fig. 5. The reminder list sent by the server is displayed on the client PDA.

In the application scenario of our system, the detection range should be from 0 to $100 \mathrm{~cm}$ or so. However, the RFID tags we used have a maximum detection distance of $10 \mathrm{~cm}$. Thus in our tests, we had to put all the object tags close to the reader one by one. This can be corrected in real applications by using a doorway RFID portal. The object data along with their corresponding RFID tag IDs are saved in a database built by MS SQL Server 2005. With this setting, the objects taken by the user can be automatically detected. The PDA then transmits the detected RFID tag IDs to the reminder server via a wireless LAN. After a reminder list is generated, the server sends the list to the client PDA carried by the user (Fig. 5). The client PDA used in this research is HP iPAQ hx7400 with an Intel PXA270 624MHz processor. The operating system on the client PDA is Microsoft Windows Mobile 2003 Second Edition.

On the server side, the system was developed by Visual Studio 2005 C\#. There are three user interfaces for system administration. The first one is for rule testing. The system administrator can select objects from the database for testing the correctness of the rules. Tag IDs from the reader PDA are not needed. The second one is for database management, including user data, object data and rule sets. The third one is for server management including initialization of the server and the communication between the server and the two PDAs (the reader PDA and the client PDA).

Experiments have been carried out with our prototype system. The user was assumed to be a school kid. A class schedule and some events were input into the system. Objects related to the classes as well as other personal belongings for daily activities were attached with an RFID tag. We then simulated the "going out" actions for one week. With the collected data, the objects are classified into one of the three frequency levels. The system then started to generate the reminder list to the user in the subsequent tests. In Fig. 5, when the user pushes the "Listening" button on the client PDA, the PDA receives and displays the reminder list generated by the server. Three objects and two object classes are displayed in one of our tests. The first three items are crayons, badminton racquet, and English textbook; the last two are sports goods and English books. The user can give a feedback on any of the reminding items by pushing the 


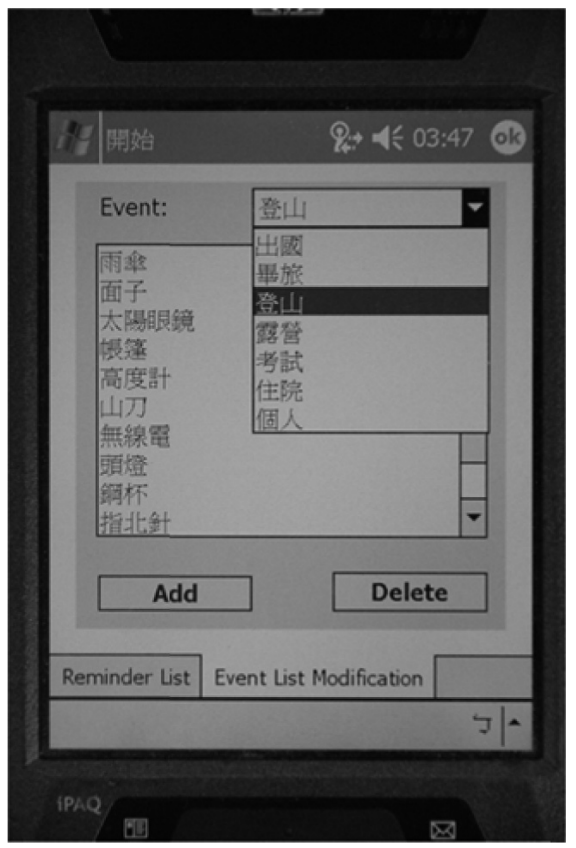

Fig. 6. Event objects editing.

"Ignore" button. The item will not appear in the same situation next time. In Fig. 6, the user can add or delete objects for an event on the client PDA. Objects for mountain climbing are shown in this example.

Generally speaking, our system could provide a personalized service in reminding the user what he/she had forgotten. The system is adaptive to the user's need. When the class schedule is changed for a new semester, it can be updated in the calendar. Also, the user can use the feedback mechanism to improve the system performance in reminding. However, in our tests, if the user brought things in an irregular manner, the system would not be able to provide useful reminder. In our experiments, the user was assumed to be a school kid. For further tests, people of different backgrounds and working professions should be considered. Next, in the current system, it can provide reminders to the user at weekends, but national holidays are not considered. Holidays can be treated as special events in the calendar. Moreover, the user may go out more than one time during a day. Time stamps can be added to the records of each "going out" and the system can analyze the user behavior not just based on the day of the week, but also the time of the day. Finally, the system can combine with weather forecast and event news from the Web. If the weather forecast says that the possibility of rain is very high this afternoon, the system can remind the user to take an umbrella with him/her. For example, if there are special discounts for members in a department store, the system can remind the user to bring the membership card.

\section{Conclusion}

A smart reminding mechanism is designed and presented in this paper. It utilizes RFID and mobile technologies. RFID provides an easy way to record the objects that the user takes out. Through the analysis on these history data, the system knows what the user should bring along on that specific day of the week. The analysis is based on the taken-out frequency of an object or a class of objects and a set of object/class rules. The event calendar is another source of information for determining what the user 
should bring along with. The objects for each event can be edited by the user or automatically generated by the system from the detected object list. After sensing the objects the user brings along with, the server constructs a reminder list with objects and classes and sends it to the client PDA or mobile phone carried by the user. The user then knows what items he/she might have forgotten. A prototype system has been developed in this research. With further testing and fine tuning, the system should be applicable to daily living.

\section{References}

[1] V.B. Misic and J.V. Misic, Improving sensing accuracy in cognitive PANs through modulation of sensing probability, Mobile Information Systems 5 (2009), 177-193.

[2] W. Wu, X. Li, S. Xiang, H.-B. Lim and K.-L. Tan, Sensor relocation for emergent data acquisition in sparse mobile sensor networks, Mobile Information Systems 6 (2010), 155-176.

[3] E.M. Shakshuki, X. Xing and T.R. Sheltami, Fault reconnaissance agent for sensor networks, Mobile Information Systems 6 (2010), 229-247.

[4] H.-H. Hsu and C.-C. Chen, RFID-based human behavior modeling and anomaly detection for elderly care, Mobile Information Systems 6 (2010), 341-354.

[5] L. Jing, Z. Cheng, M. Kansen, T. Huang and S. Sun, An educational schoolbag system for providing an object reminder service, IPSJ Digital Courier 3 (2007), 64-74.

[6] Enjoy RFID technology. Obtained through the Internet: http://enjoyrfid.blogspot.com/, [accessed Feb. 7, 2011$].$

[7] J. Jayaputera and D. Taniar, Data retrieval for location-dependent queries in a multi-cell wireless environment, Mobile Information Systems 1 (2005), 91-108.

[8] A. Waluyo, B. Srinivasan and D. Taniar, Research on location-dependent queries in mobile databases, International Journal of Computer Systems: Science and Engineering 20 (2005), 79-95.

[9] A.B. Waluyo, D. Taniar, W. Rahayu and B. Srinivasan, Mobile service oriented architectures for NN-queries, Journal of Network and Computer Applications 32 (2009), 434-447.

[10] A.B. Waluyo, D. Taniar, W. Rahayu and B. Srinivasan, Mobile broadcast services with MIMO antennae in 4G wireless networks, World Wide Web Journal (2011). (DOI: 10.1007/s11280-011-0113-9, online for early access).

[11] A.B. Waluyo, W. Rahayu, D. Taniar and B. Srinivasan, A novel structure and access mechanism for mobile broadcast data in digital ecosystems, IEEE Transactions on Industrial Electronics, (2010). (DOI: 10.1109/TIE.2009.2035457, online for early access).

[12] Microsoft Home Virtual Presskit. Obtained through the Internet: http://www.microsoft.com/presspass/events/mshome/ default.mspx, [accessed Feb. 7, 2011].

[13] Toyota Home. Obtained through the Internet: http://www.toyotahome.co.jp/, [accessed Feb. 7, 2011].

[14] RFID Journal. Obtained through the Internet: http://www.rfidjournal.com/, [accessed Feb. 7, 2011].

[15] J. Bohn, Prototypical implementation of location-aware services based on a middleware architecture for super-distributed RFID tag infrastructures, Proceedings 19th International Conference on Architecture of Computing Systems (ARCS06), 2006, pp. 155-166.

[16] Y. Nakauchi, T. Suzuki, A. Tokumasu and S. Murakami, Cooking procedure recognition and support system by intelligent environments, Proceedings IEEE Workshop on Robotic Intelligence in Informationally Structured Space (RIISS '09), 2009, pp. 99-106.

[17] M. Darianian and M.P. Michael, Smart home mobile RFID-based Internet-of-things systems and services, Proceedings 2008 International Conference on Advanced Computer Theory and Engineering, 2008, pp. 116-120.

[18] E. Becker, V. Metsis, R. Arora, J. Vinjumur, Y. Xu and F. Makedon, SmartDrawer: RFID-based smart medicine drawer for assistive environments, Proceedings 2nd International Conference on PErvsive Technologies Related to Assistive Environments (PETRA'09), 2009.

[19] M. Stikic, T. Huỳnh, K.V. Laerhoven and B. Schiele, ADL recognition based on the combination of RFID and accelerometer sensing, Proceedings Second International Conference on Pervasive Computing Technologies for Healthcare (PervasiveHealth 2008), 2008, pp. 258-263.

[20] S. Im, I.J. Kim, S.C. Ahn and H.G. Kim, Automatic ADL classification using 3-axial accelerometers and RFID sensor, Proceedings IEEE International Conference on Multisensor Fusion and Integration for Intelligent Systems (MFI 2008), 2008, pp. 697-702.

[21] D. Cook and S. Das, Smart Environments: Technology, Protocols and Applications, Wiley-Interscience, 2005.

[22] R. Kadouche, B. Abdulrazak, M. Mokhtari, S. Giroux and H. Pigot, A semantic approach for accessible services delivery in a smart environment," International Journal of Web and Grid Services 5 (2009), 192-218. 
[23] B. Abdulrazak, B. Chikhaoui, C. Gouin-Vallerand and B. Fraikin, A standard ontology for smart spaces, International Journal of Web and Grid Services 6 (2010), 244-268.

[24] V.R. Jakkula, A.S. Crandall and D.J. Cook, Knowledge discovery in entity based smart environment resident data using temporal relation based data mining, Proceedings Seventh IEEE International Conference on Data Mining Workshops (ICDMW 2007), 2007, pp. 625-630.

[25] P. Rashidi and D.J. Cook, Keeping the intelligent environment resident in the loop, Proceedings 4th International Conference on Intelligent Environments, (IE 2008), 2008, pp. 1-9.

[26] D.J. Cook, M.S. Edgecombe, A. Crandall, C. Sanders and B. Thomas, Collecting and disseminating smart home sensor data in the CASAS project, Proceedings CHI Workshop on Developing Shared Home Behavior Datasets to Advance HCI and Ubiquitous Computing Research, 2009.

[27] O. Brdiczka, J.L. Crowley and P. Reignier, Learning situation models in a smart home, IEEE Transactions on Systems, Man and Cybernetics - Part B 39 (2009), 56-63.

[28] H. Si, S.J. Kim, N. Kawanishi and H. Morikawa, A context-aware reminding system for daily activities of dementia patients, Proceedings 27th International Conference on Distributed Computing Systems Workshops (ICDCSW'07), 2007, p. 50.

Hui-Huang Hsu is an Associate Professor in the Department of Computer Science and Information Engineering at Tamkang University, Taipei, Taiwan. He received his PhD and MS Degrees from the Department of Electrical and Computer Engineering at the University of Florida, USA, in 1994 and 1991, respectively. He has published over 90 referred papers and book chapters, as well as participated in many international academic activities. His current research interests are in the areas of machine learning, data mining, ambient intelligence, bio-medical informatics, and multimedia processing. Dr. Hsu is a senior member of the IEEE.

Cheng-Ning Lee received his MS Degree from the Department of Computer Science and Information Engineering at Tamkang University, Taipei, Taiwan in 2010. His research interests include mobile computing and RFID applications.

Jason C. Hung is an Associate Professor of Department of Information Management at Overseas Chinese University, Taiwan, ROC. His research interests include Multimedia Computing and Networking, Distance Learning, E-Commerce, and Agent Technology. From 1999 to date, he was a part time faculty of the Computer Science and Information Engineering Department at Tamkang University. Dr. Hung received his BS and MS degrees in Computer Science and Information Engineering from Tamkang University, in 1996 and 1998, respectively. He also received his Ph.D. in Computer Science and Information Engineering from Tamkang University in 2001. Dr. Hung has published over 70 papers and book chapters, as well as participated in many international academic activities, including the organization of many international conferences. He is the founder and Workshop chair of International Workshop on Mobile Systems, E-commerce, and Agent Technology. He is also the Associate Editor of the International Journal of Distance Education Technologies, published by Idea Group Publishing, USA. Web: http://www.ocu.edu.tw / jhung.

Timothy K. Shih is a Professor of Department of Computer Science and Information Engineering, National Central University, Taiwan. He is a Fellow of the Institution of Engineering and Technology (IET). In addition, he is a senior member of ACM and a senior member of IEEE. He has edited many books and published over 430 papers and book chapters. He was the founder and co-editor-in-chief of the International Journal of Distance Education Technologies, published by Idea Group Publishing, USA. $\mathrm{He}$ is an Associate Editor of the ACM Transactions on Internet Technology and an associate editor of the IEEE Transactions on Learning Technologies. He was also an Associate Editor of the IEEE Transactions on Multimedia. He has received many research awards, including research awards from National Science Council of Taiwan, IIAS research award from Germany, HSSS award from Greece, Brandon Hall award from USA, and several best paper awards from international conferences. 

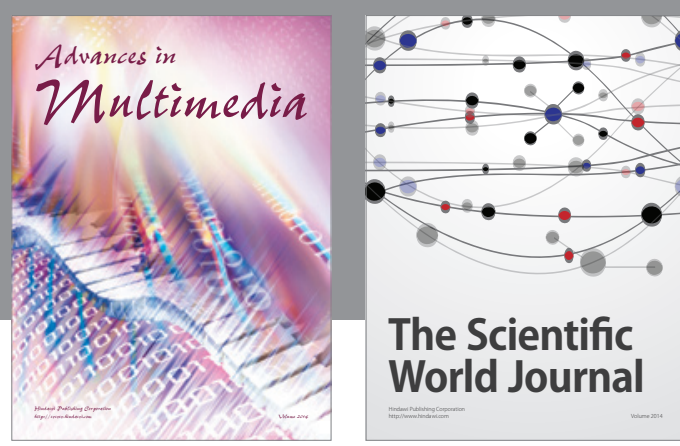

The Scientific World Journal
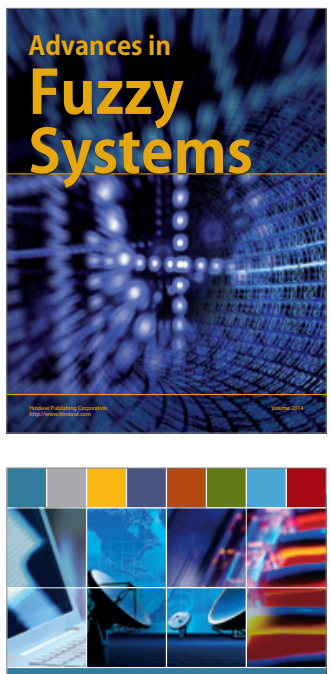

Computer Networks and Communications
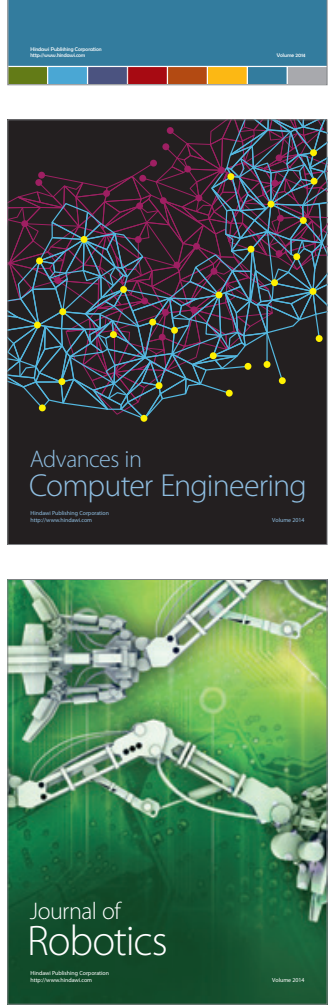
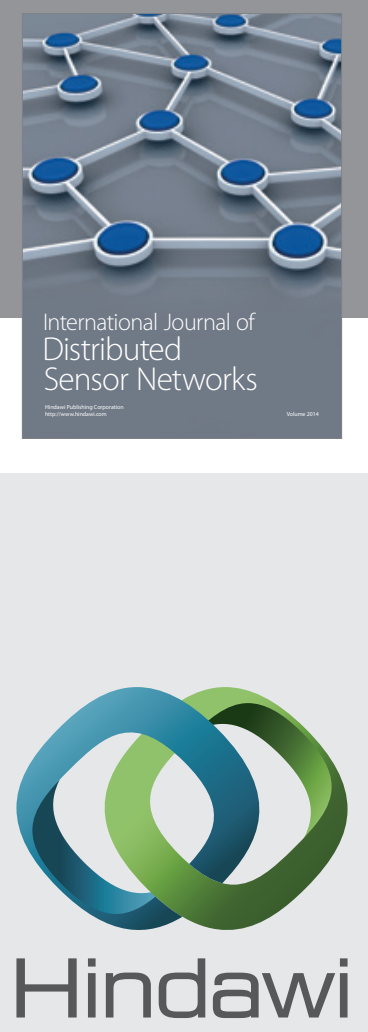

Submit your manuscripts at

http://www.hindawi.com
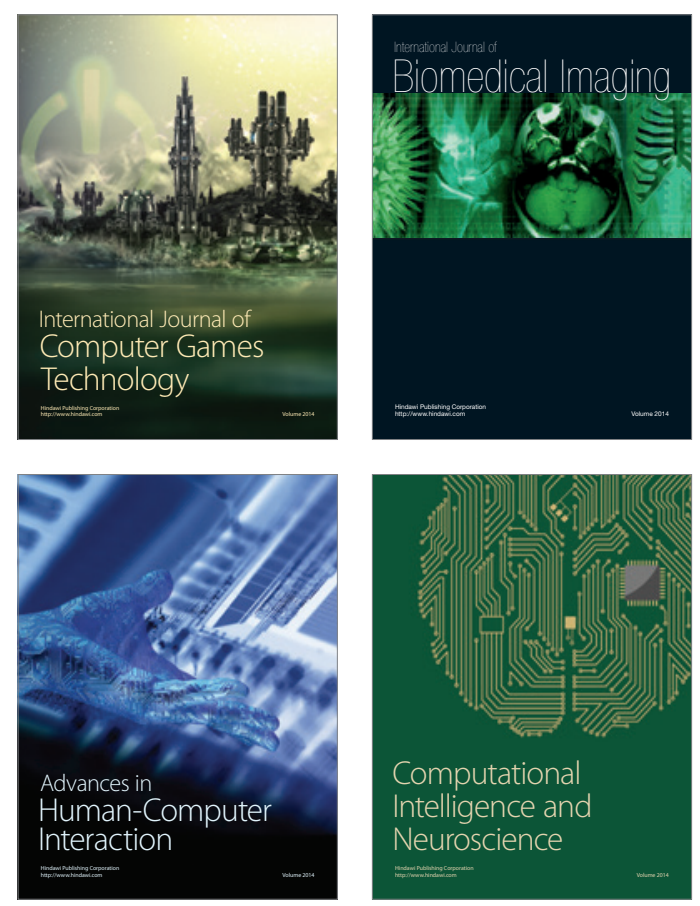
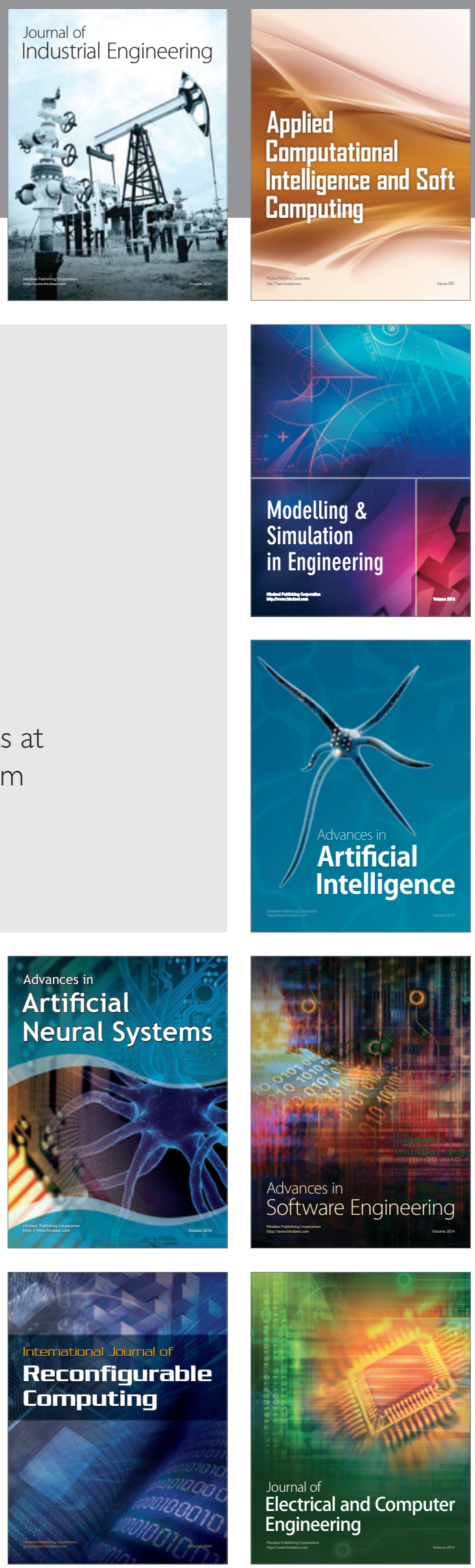